Original Paper http://ajol.info/index.php/ijbcs http://indexmedicus.afro.who.int

\title{
Typologie de la pêche de la langouste verte Panulirus regius, De Brito Capello, 1864 sur le littoral de la Côte d'Ivoire (Afrique de l'Ouest)
}

\author{
Ida Akissi KONAN ${ }^{*}$, Olivier Assoi ETCHIAN ${ }^{1}$, Basile Kouakou KOUAME ${ }^{1}$, \\ Jean-Noel YAPI ${ }^{1}$, Angelina Gbohono LOUKOU ${ }^{1}$ et Vincent KADJO ${ }^{2}$ \\ ${ }^{1}$ Laboratoire de Biologie et Cytologie Animales, UFR-SN, Université Nangui Abrogoua, 02 BP 801 Abidjan \\ 02, Côte d'Ivoire. \\ ${ }^{2}$ Département de Biologie Animale, Université Peleforo Gon Coulibaly, BP 1328 Korhogo, Côte d'Ivoire. \\ *Auteur correspondant ; E-mail: idaakissikonan@gmail.com
}

Received: 07-04-2021

Accepted: $20-08-2021$

Published: 31-08-2021

\section{RESUME}

En Côte d'Ivoire, la langouste fait partir des crustacés présents dans les captures de la pêche maritime artisanale. Cependant, aucune donnée scientifique n'est disponible quant aux acteurs de la pêche de cette protéine. Ainsi, la présente étude a pour objectif de caractériser les acteurs de la pêche à la langouste verte Panulirus regius (De Brito Capello, 1864) pratiquée au niveau de la Zone Economique Exclusive de la Côte d'Ivoire. L'étude a nécessité une enquête le long du littoral sur un effectif de 60 pêcheurs de langouste. Les résultats ont montré que la pêche langoustière est pratiquée essentiellement par les Ivoiriens et les Ghanéens. De plus, la tranche d'âge dominante chez ces pêcheurs est comprise entre 30 et 45 ans chez les Ivoiriens et Ghanéens avec des proportions de $20 \%$ et $25 \%$, respectivement. Au niveau de la quantité de la langouste verte pêchée, la grande saison froide a enregistré la plus grande quantité de langoustes capturées.

(C) 2021 International Formulae Group. All rights reserved.

Mots clés : Langoustes vertes, Panulirus regius, Acteurs de pêche, Zone Economique Exclusive, Côte d'Ivoire.

\section{Fishing typology of the green lobster Panulirus regius, De Brito Capello, 1864 along the coast of Côte d'Ivoire (West Africa)}

\begin{abstract}
In the Côte d'Ivoire, lobster is part of the crustaceans present in the catches of artisanal maritime fishing. However, no scientific data is available on the actors of the fishery of this protein. Thus, the objective of this study is to characterize the actors of the green lobster Panulirus regius (De Brito Capello, 1864) fishery practised in the Exclusive Economic Zone of the Côte d'Ivoire. The study required a survey along the coastline of 60 lobster fishermen. The results showed that green lobster fishing is mainly practised by citizens of Côte d'Ivoire and Ghana. Fishermen who practised this activity were aged between 30 and 45 years old with percentages of $20 \%$ and $25 \%$ for citizens of Côte d'Ivoire and Ghana, respectively. In terms of the quantity lobster captured, the largest quantity was recorded on the great cold season.

(C) 2021 International Formulae Group. All rights reserved.
\end{abstract}

Keywords: Green (spiny) lobsters, Panulirus regius, Fishing actors, Exclusive Economic Zone, Côte d'Ivoire. 


\section{INTRODUCTION}

Panulirus regius De Brito Capello, 1864 (la langouste verte ou royale) est connue de la Méditerranée occidentale de la côte ouest-africaine, du Rio de Oro à l'Angola (Crosnier, 1971 ; Maigret, 1978). Selon ces auteurs, $P$. regius est la seule langouste rencontrée dans le sud du golfe de Guinée. D'un point de vue économique, c'est l'espèce de crustacés la plus importante trouvée en Afrique de l'Ouest (Freitas et al., 2007). Ce crustacé côtier est pêché au chalut avec un maximum de concentration vers 8-10 m de profondeur (Maigret, 1978) et en faible quantité à 25-30 m de profondeur (Dia et al., 2015). Cette espèce vit dans les eaux côtières agitées et continuellement remuées. Elle se rencontre dans les zones rocheuses pendant la journée où elle s'abrite dans les anfractuosités ; la nuit, elle sort sur les fonds sableux pour se nourrir. $P$. regius est une espèce aussi rencontrée en grand nombre à côté des zones à épave (Dia et al., 2015).

En Côte d'Ivoire, P. Regius est une espèce exploitée presqu'exclusivement par la pêche artisanale. Elle constitue une source non négligeable de protéine animale pour les populations Ivoiriennes et de revenu pour les populations côtières. Elle a par ailleurs une excellente valeur nutritionnelle et organoleptique (FAO, 2018). Cependant, la filière de la langouste est peu connue par la communauté scientifique Ivoirienne et manque de base de données disponible pour une prise en main de sa gestion. Au vu de la présence régulière de la langouste dans les captures de la pêche artisanale maritime, il devient nécessaire voir même indispensable de se pencher sur cette filière afin d'établir une base de données conséquente pour une vulgarisation de la filière. Cela passe nécessairement par la connaissance des acteurs d'où l'intérêt de cette étude. Ainsi l'objectif de cette étude vise à dresser le portait des acteurs impliqués dans cette pêche langoustière artisanale au cours des différentes saisons marines et aussi d'estimer la quantité de cette ressource capturée sur le littoral de la Côte d'Ivoire.

\section{MATERIEL ET METHODES}

\section{Milieu d'étude}

L'étude a été réalisée le long du littoral Ivoirien sur une période de 12 mois, allant de septembre 2019 à août 2020. Quatre localités ont été retenues pour les enquêtes notamment, les localités d'Assinie, Grand Bassam, Canal de Vridi et Sassandra. Le choix des sites a été fait en fonction de la présence effective des pêcheurs de langouste sur toute la période de l'étude. La Figure1 représente la localisation géographique des différents sites d'enquêtes.

\section{Echantillonnage et traitement des données}

À l'issue d'une enquête préliminaire (juin 2019 à août 2019), les données collectées ont permis d'élaborer le questionnaire d'enquête. La collecte des données a consisté à des entretiens individuels avec les pêcheurs ayant pêché au moins une fois la langouste. L'enquête s'est déroulée sur une période d'un (1) an allant de septembre 2019 à août 2020. Un ensemble de 60 pêcheurs ont été interrogés dont 23 à Grand Bassam, 11 à Assinie, 5 à Vridi canal et 21 à Sassandra. Les interrogations ont porté sur la nationalité, la situation matrimoniale, l'âge, la spécialité dans la pêche, la profondeur de pêche, les mois de pêche, les prix de vente, la quantité de langouste pêchée par sortie, la technique de pêche, les outils de pêche et la zone de pêche. Les données obtenues ont été traitées avec l'outil tableau croisé dynamique du logiciel Excel. Les proportions des ethnies impliquées dans la pêche langoustière, leur répartition par âge selon la nationalité et leur situation matrimoniale ont été traitées à l'aide d'un test $\mathrm{G}$ (équivalent du test Khi deux ( $\chi 2)$ ). Le test $\mathrm{t}$ de Student a permis de comparer les quantités de langoustes capturées entre les localités d'Assinie, Grand Bassam, Canal de Vridi et Sassandra. Le logiciel $\mathrm{R}$ version 2.10 .1 a servi à la réalisation de ces tests. Le seuil de signification de ces tests est $\alpha=0,05$. 


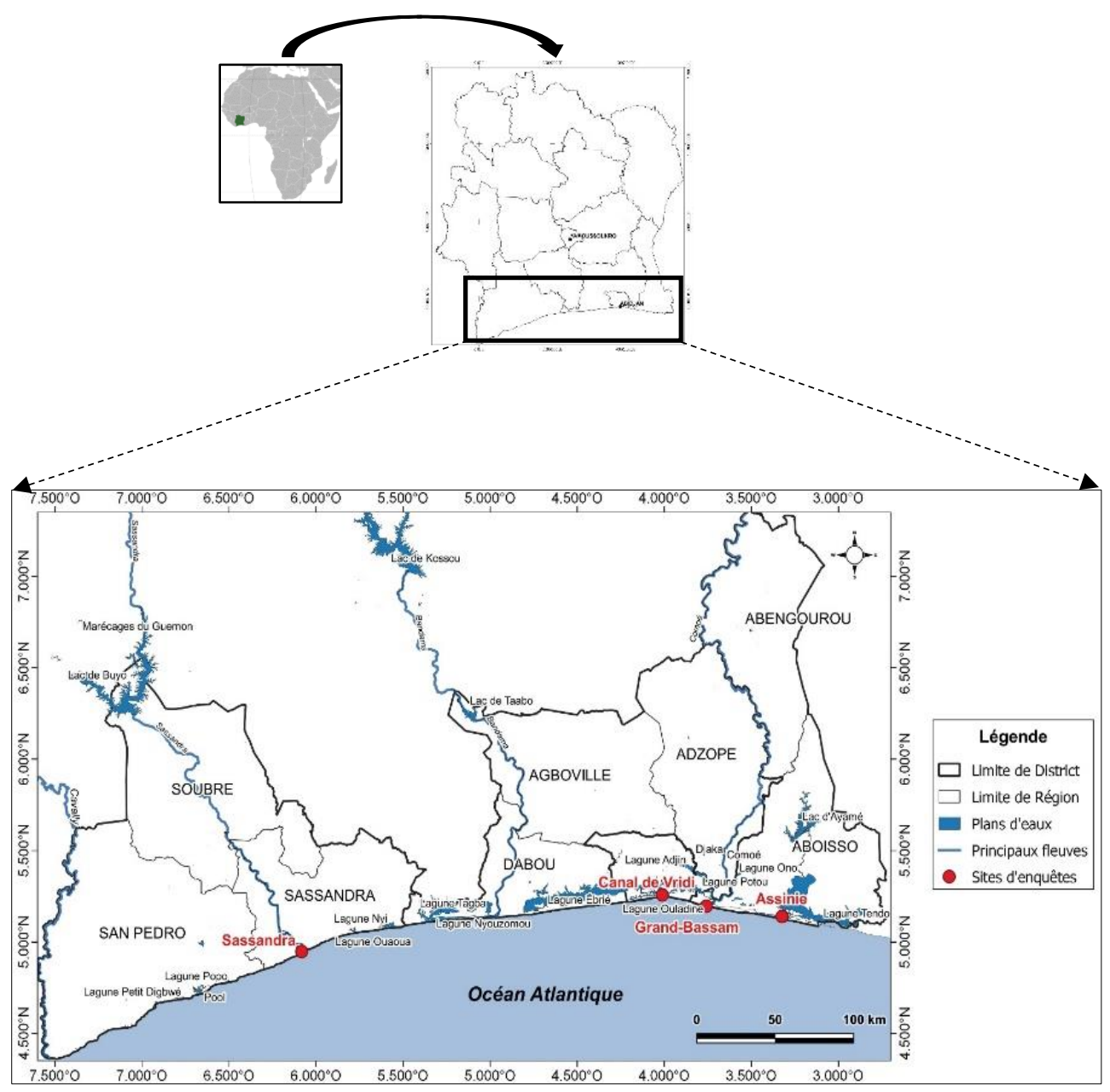

Figure 1 : Localisation géographique des différents sites d'enquêtes.

\section{RESULTATS}

\section{Nationalité des pêcheurs}

La Figure 2 fait état des nationalités présentes dans l'activité de pêche langoustière en Côte d'Ivoire. Les résultats ont montré que des quatre localités, Grand-Bassam et Sassandra sont celles qui ont enregistré les plus grands nombres de pêcheurs avec les effectifs respectifs de 23 et 21 pêcheurs. Aussi, l'activité de pêche est exclusivement occupée par deux nationalités dont les Ivoiriens et les Ghanéens. Mais, contrairement au Ivoiriens qui sont présents seulement au niveau des localités d'Assinie et Grand-Bassam, les Ghanéens se rencontrent partout notamment, à Assinie, Grand-Bassam, Sassandra et Vridi Canal. Au niveau de Grand-Bassam, la proportion des pêcheurs Ivoiriens $(86,96 \%)$ dominent significativement celle des pécheurs Ghanéens $(\mathrm{P}<0,05)$. Au contraire, les pêcheurs Ghanéens sont plus nombreux avec un taux de participation de $81,82 \%$ à Assinie.

\section{Situation matrimoniale des pêcheurs}

Cette étude enregistre la présence de célibataires et de mariés (Figure 3) dans la pratique de l'activité de pêche de la langouste. En effet, l'enquête enregistre dans l'ensemble, $85 \%$ de pêcheurs mariés avec dominance des Ghanéens (56,67\%) de plus de deux (2) fois sur les Ivoiriens $(\mathrm{p}<0,05)$. Sur les $15 \%$ de célibataires, $8,33 \%$ sont Ivoiriens et $6,67 \%$ sont 
étrangers. Cependant, aucune différence notable n'est enregistrée entre les célibataires de ces deux peuples $(p>0,05)$.

\section{Répartition par classes d'âge}

Trois classes d'âge se dégagent de l'âge des pêcheurs de la langouste verte au niveau des deux nationalités $(\leq 30]$;] $30 ; 45]$ et $>45)$ (Figure 4). La tranche d'âge dominante est celle des pêcheurs d'âge compris entre 30 et 45 ans aussi bien chez les nationaux que les non nationaux avec un taux de $45 \%$ de l'ensemble des pêcheurs. Dans cette tranche, les nationaux renferment $20 \%$ contre $25 \%$ chez les Ghanéens. Ensuite, vient la classe d'âge des pêcheurs d'âge inférieur ou égale à 30 ans avec un taux de participation de $36,67 \%$. Cependant, dans cette classe d'âge, la proportion des étrangers (23,33\%) est de 1,75 fois supérieure à celle des Ivoiriens. Enfin, la classe d'âge des pêcheurs âgés de plus de 45 ans enregistre une proportion d'étrangers (15\%) de 4,5 fois supérieures à celle des Ivoiriens pour la même tranche d'âge.

\section{Quantités de langoustes pêchées}

La Figure 5 présente les quantités de langouste pêchée au cours des différentes saisons marines. Il ressort de l'analyse de la figure que des quatre (4) saisons marines, c'est la grande saison froide qui enregistre la plus grande quantité de langouste pêchée (7326 $\mathrm{Kg}$ ). La quantité pêchée au cours de cette saison est respectivement de 1,$9 ; 2,2$; et 8,9 fois plus grande que celle de la petite saison froide (PSF), la grande saison chaude (GSC) et la petite saison chaude.

\section{Difficultés rencontrées par les pêcheurs de langouste}

Les pêcheurs des différentes localités sont en majorité confrontés aux mêmes difficultés notamment, le coût élevé des filets de pêche pour langouste, la perte des filets de pêche, accrochés le plus souvent par les chalutiers pratiquant la pêche industrielle lors de leur passage dans les espaces de pêche de langouste. Une autre difficulté est l'effort de pêche énorme, dû à l'important effort physique fourni pour contenir les courants marins. A Grand-Bassam, la destruction des pirogues par les vagues est très récurrente, rendant l'activité de pêche plus difficile dans cette zone. Au niveau de la commercialisation de la langouste, les pêcheurs d'Assinie se plaignent des mareyeurs qui achètent la langouste à faible prix (5000f) et revendent aux détaillants à un prix plus élevé (9000f à $10000 f \mathrm{le} \mathrm{kg}$ ).

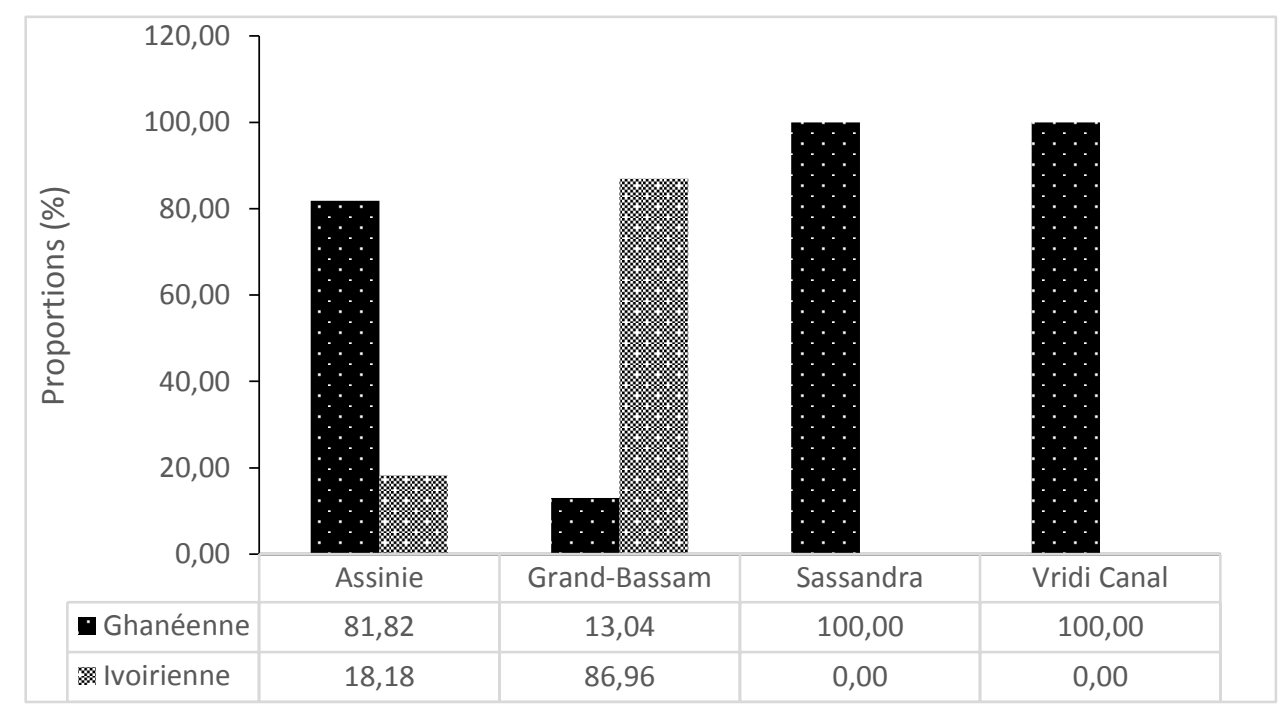

Figure 2 : Nationalité des pêcheurs de langouste des différentes localités d'étude $(\mathrm{N}=60)$. 


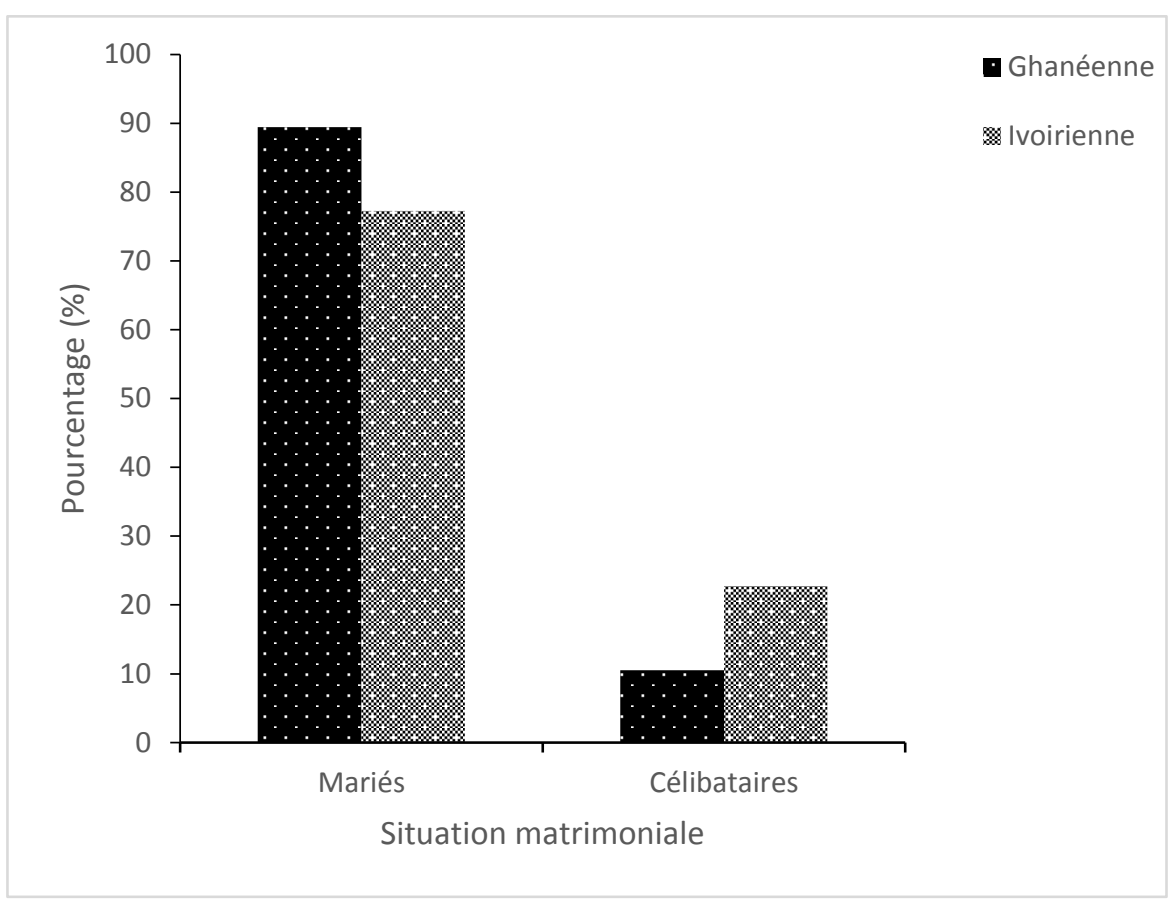

Figure 3 : Situation matrimoniale des pêcheurs de langouste de la zone d'étude $(\mathrm{N}=60)$.

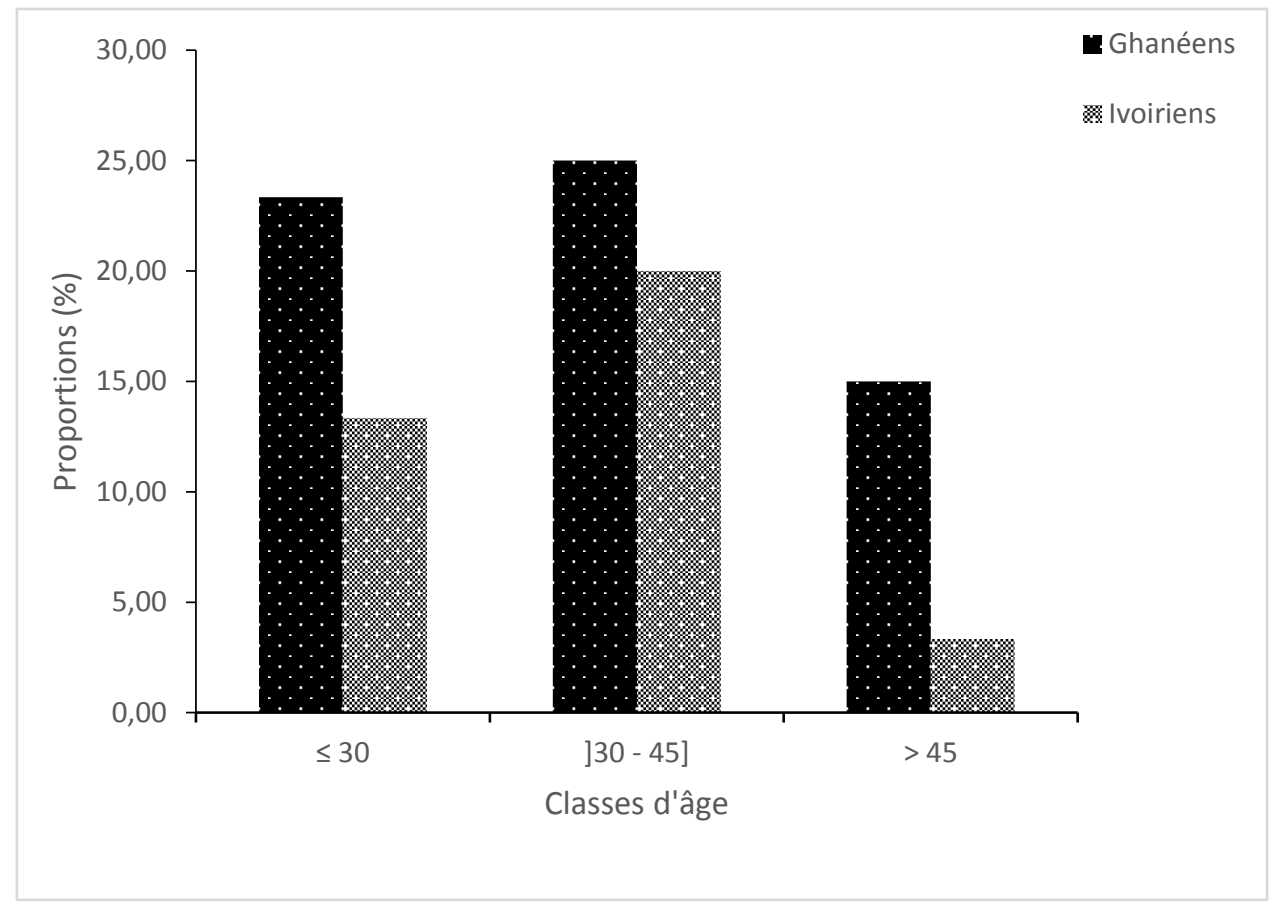

Figure 4 : Répartition par classes d'âge des pêcheurs de la langouste verte. 


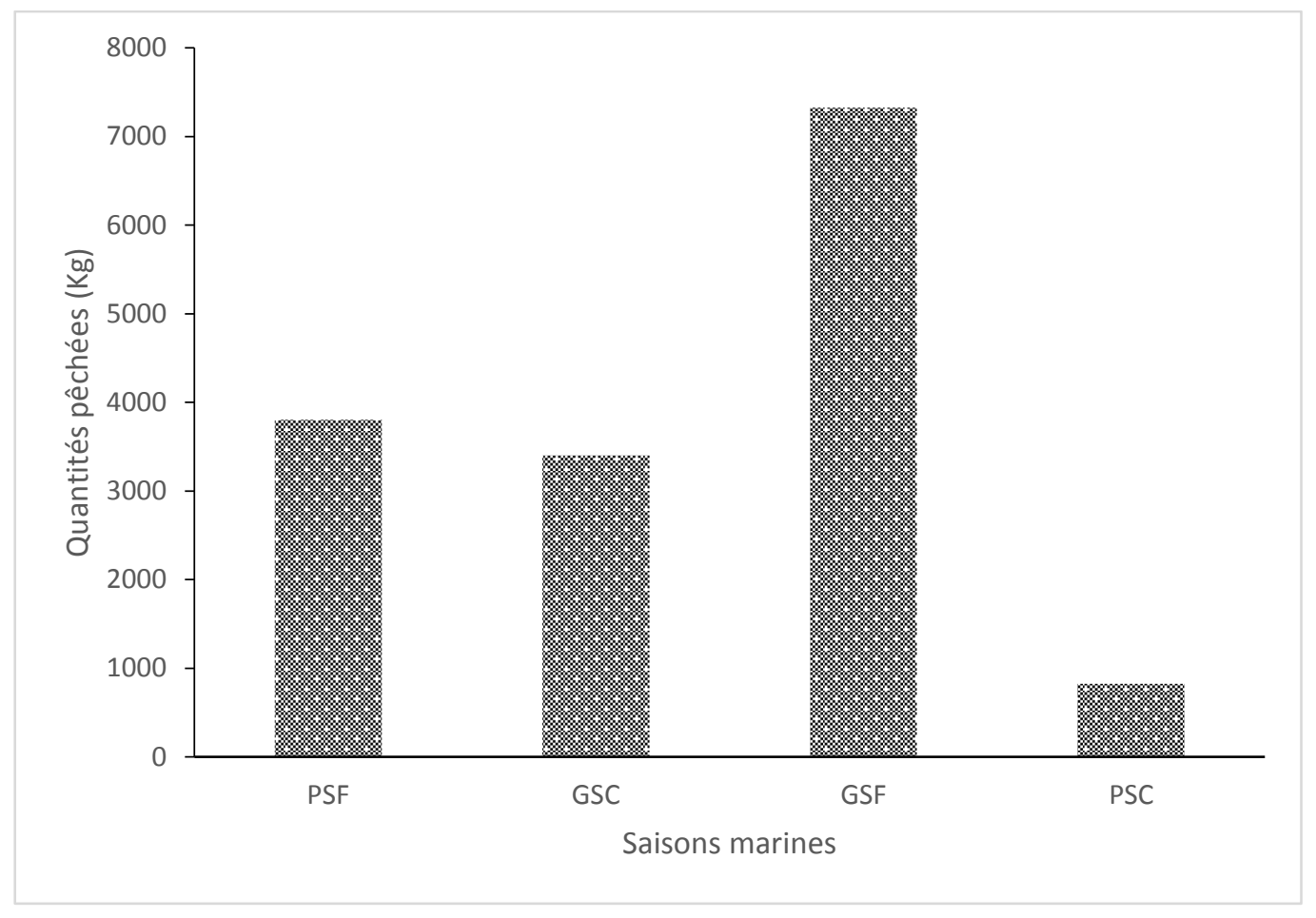

Figure 5 : Quantités de langoustes pêchées au cours des différentes saisons marines.

\section{DISCUSSION}

Les résultats de cette enquête ont montré qu'en Côte d'Ivoire, la pêche aux langoustes est essentiellement pratiquée par les Ivoiriens et les Ghanéens. Cependant, une nette dominance des Ghanéens est enregistrée bien qu'au niveau de Grand-Bassam, les Ivoiriens soient les plus nombreux. La dominance des Ghanéens sur les Ivoiriens serait due au fait que la pêche des langoustes se pratique dans le milieu marin. En effet, contrairement aux Ivoiriens qui sont beaucoup présents au niveau de la pêche lagunaire, les Ghanéens pratiquent plus au niveau de la pêche maritime. Des travaux menés par Delaunay (1995) avaient déjà souligné que les Ghanéens étaient des professionnels de la pêche au niveau de la mer. Selon lui, ces derniers sont caractérisés par leur dynamisme et leur grand savoir-faire en matière de pêche maritime. La participation de ces quelques Ivoiriens dans l'activité pourrait non seulement être due à la cohabitation de ceux-ci avec les Ghanéens mais aussi, à la réduction des captures au niveau de la lagune. Par ailleurs, la pêche maritime demande plus effort physique et présente plus de risque par rapport à la pêche lagunaire à laquelle sont habitués les Ivoiriens. Les travaux de Koffi (1997) sur la pêche artisanale maritime s'étaient soldés par des résultats similaires. En effet, selon cet auteur, le métier de pêcheur est physiquement éprouvant et souvent dangereux et la survie des hommes dépend de leur courage. Le nombre élevé de pêcheurs mariés enregistré lors de cette étude se justifierait par les charges familiales plus élevées chez ces derniers. En effet, les pêcheurs mariés ont besoin de plus de revenu pour nourrir la famille, scolariser les enfants, etc... Cette étude révèle aussi que dans l'ensemble des localités, la grande partie des pêcheurs ne vivent que du fruit de la pêche. Ceci porterait à dire que la pêche de la langouste est une activité à fort revenu. D'ailleurs, dans un pays comme le Sénégal, les pêcheurs de langouste préfèrent ne pas scolariser leurs enfants au 
profit de l'activité compte tenu de son caractère très lucratif. L'activité de pêche de la langouste en Côte d'Ivoire est dominée par les jeunes notamment, ceux d'âge compris entre 31 et 45 ans. Cela serait dû au fait que la pêche artisanale maritime en général demande beaucoup d'effort physique à plus forte raison celle de la langouste qui se fait dans des profondeurs de plus de 10 mètres et demandant une pose de filets par plongée. Les travaux de Jaziri et al. (2014) ont donné des résultats similaires. En effet, ces auteurs ont mené une étude sur les unités d'exploitation de la langouste rouge en Tunisie et ont trouvé que 49\% des pêcheurs avaient un âge compris entre 31 et 50 ans. Les travaux de cette étude montrent par ailleurs que les plus grandes quantités de langoustes sont pêchées pendant les saisons froides. Ceci pourrait être dû au phénomène d'upwelling pendant ces saisons. En effet, ce phénomène caractérisé par la remontée des eaux froides en surface, entraîne un appauvrissement des profondeurs marines en nutriment, poussant ainsi les langoustes à parcourir plus distance pour rechercher la nourriture. Ce qui les exposerait plus aux captures des pêcheurs. De plus, ce phénomène provoque la destruction des terriers des langoustes et facilite ainsi leur capture. Cependant, les travaux de Clotilde-Ba et al. (1997) en disent le contraire. En effet, dans les travaux de ces auteurs, il ressort que les captures les plus abondantes de langouste sont observées pendant la saison chaude. Plusieurs difficultés ont été signalées dans cette activité par les pêcheurs. Ces difficultés pourraient être dues au manque d'organisation ou d'une stratégie de pêche inappropriée. En effet, en se mettant en association, les pêcheurs de langouste pourraient non seulement fixer euxmêmes des prix convenable en tenant compte des risques du métier et des charges liées à l'achat du matériel utilisé pour la pêche. Par ailleurs, ils pourraient négocier avec les autorités compétentes quant à disposer de zones de pêche ou être avertis des éventuels jours de passage des chalutiers dans la zone de pêche.

\section{Conclusion}

Cette étude monte que la pêche aux langoustes est encore à l'étape artisanale avec une présence des nationaux (N'zima) et non nationaux (Ghanéens : Fanti) dans l'activité. La pêche est dominée par des individus âgés de 30 à 45 ans. La quantité de langouste pêchée est plus importante pendant la grande saison froide. Au vu de ces résultats, il serait intéressant de mener une étude sur la dynamique des populations de cette espèce en vue d'une gestion durable du stock.

\section{CONFLIT D'INTERETS}

Les auteurs déclarent qu'aucun conflit d'intérêt ne les oppose quant à la réalisation du présent travail.

\section{CONTRIBUTIONS DES AUTEURS}

Ce projet a été initié et approuvé par $\mathrm{OAE}$ et BKK qui ont dirigé les travaux. Les travaux de terrain ont été exécutés par IAK et AGL. JNY et VK ont participé l'analyse des données et la rédaction du manuscrit.

\section{REMERCIEMENTS}

Les auteurs tiennent à remercier tous ceux qui ont facilité les travaux de terrain en particulier M. N'Guessan et M. Kouamé pour la collecte des langoustes à Assinie.

\section{REFERENCES}

Clotilde-Ba FL, Diatta Y, Capapé C. 1997. Observation sur huit espèces comestibles de crustacés décapodes des eaux sénégalaises (Afrique de l'ouest). Laboratoire de Biologie Marine, Département de Biologie Animale, Université cheikh Anta Diop de Dakar, Sénégal. Bolletim do Museu Municipal do Funchal, 49(282) :171-187.

Crosnier A. 1971. Ponte et développement larvaire de la langouste verte Panulirus regius de Britto Capello, 1864 dans le sud du Golfe de Guinée. Cahier ORSTOM, série Océanographie, 9(3) : 339-361.

Delaunay K. 1995. Les pêcheurs Ghanéens (Fante et Ewe) sur le littoral ivoirien. Histoire de la pêche piroguière maritime en Côte-d'Ivoire au XXe siècle. Thèse du 
doctorat des Sciences Humaines Université de Paris 1, PanthéonSorbonne, $574 \mathrm{p}$.

Dia MA, Kamara A, Sow AH, Ba SA. 2015. Biométrie et Éléments de Biologie de la Langouste verte (Panulirusregius, De Brito Capello, 1864) des côtes de Nouadhibou (Mauritanie). Bulletin de la Société Zoologie de France., 140 (1):6177.

FAO. 2018. La situation mondiale des pêches et de l'aquaculture. Atteindre les objectifs de développement durable. Rome. Licence : CC BY-NC-SA 3.0 IGO, 254 p.

Freitas R, Medina A, Correia S, Castro M. 2007. Reproductive biology of spinylobsterPanulirus regius from the northwestern Cape Verde Islands. African Journal of Marine Science, 29(2): 201208.

DOI:

10.2989/AJMS.2007.29.2.5.188.
Jaziri S, Ben Salem S, GaamourA. 2014. L'analyse technico-économique des unités d'exploitation de la langouste rouge dans le port de pêche de Bizerte. Institut National des Sciences et Technologies de la Mer, Centre La Goulette, Tunisie, 41 : 10 p.

Koffi BCY. 1997. La pêche artisanale maritime en Côte d'Ivoire: étude géographique. Thèse de Doctorat. Université de Nantes, France, $324 \mathrm{p}$.

Maigret J.1978. Contribution à l'étude de la Langouste verte de la côte occidentale d'Afrique. Thèse de Doctorat ès-Sciences Naturelles. Université d'Aix-Marseille, $264 \mathrm{p}$.

Marchal E, Barro M. 1964. Contribution à l'étude de la langouste verte africaine Panulirus"rissoni Desmarest 1825 ( $P$. regius De Brito Capello). Cahiers de l'ORSTOM, série Océanographie, 2 (3) : 57-69. 